\title{
Wolf-Rayet binaries: old friends and new acquaintances
}

\author{
Virpi S. Niemela ${ }^{1,2,3}$, Roberto Gamen ${ }^{2,4}$, Nidia I. Morrell ${ }^{2,5}$, \\ and Sixto Giménez Benítez ${ }^{2,4}$ \\ Facultad de Ciencias Astronómicas y Geofísicas, \\ U.N. La Plata, Paseo del bosque s/n, 1900 La Plata, Argentina
}

\begin{abstract}
Observations of WR stars in binary systems are discussed, emphasizing constraints on our knowledge of the binary frequency of WR stars, and of WR stars as a distinctive class of objects. Radial velocity orbits of newly discovered binaries, e.g., WR 29, a short period WN7+OB binary in our Galaxy, and $\mathrm{SMC} / \mathrm{AB} 7$, a massive $\mathrm{WN}+\mathrm{O} 7$ binary in the Small Magellanic Cloud, are presented. New spectroscopic observations of binary systems with previously known orbits are also reported, showing in the case of WR 21 evidence of change of the orbital elements as derived from different spectral lines. An elliptic orbit for CV Ser is also illustrated.
\end{abstract}

\section{About the frequency of binaries among Wolf-Rayet stars}

There are 218 Population I Wolf-Rayet stars at present known in our Galaxy, according to the new catalogue (van der Hucht 1999 and these Proceedings). How many of these may be binary systems? Information about the binary nature of WR stars in our Galaxy exists for approximately 40 stars. This information includes:

(a) Spectroscopic binaries with known orbits. Both double-lined and single lined binaries are known;

(b) Visual binaries, i.e., visually double stars with additional information indicating that they are physically bound. Examples of this class, are WR 146 and WR 147 (WR numbers from van der Hucht et al. 1981) observed as visual binaries with HST (Niemela et al. 1998). In addition, WR 146 and WR 147 are also sources of non thermal radiation arising from the colliding winds of their components ( $c f$. Williams et al. 1997; Dougherty et al. 1996), thus indicating they are physical binaries;

(c) Variable radial velocity of spectral lines in WR+OB type spectra. When the binary period is not known, a diagram indicating opposite variations for the

\footnotetext{
${ }^{1}$ Visiting Astronomer, CTIO, NOAO, operated by AURA, Inc. under cooperative agreement with the NSF

${ }^{2}$ Visiting Astronomer, CASLEO, operated under agreement between CONICET, SeCyT, and the Universities of La Plata, Córdoba and San Juan, Argentina

${ }^{3}$ Member of Carrera del Investigador, CIC, Prov. Buenos Aires, Argentina

${ }^{4}$ Fellow of CIC, Prov. Buenos Aires, Argentina

${ }^{5}$ Member of Carrera del Investigador, CONICET, Argentina
} 
Table 1. Galactic Wolf-Rayet stars with $v<10 \mathrm{mag}$

\begin{tabular}{|c|c|c|c|c|c|}
\hline WR & $\mathrm{HD} /$ name & $v^{a}$ & spectral type $^{a}$ & binary status ${ }^{h, i}$ & remarks \\
\hline 6 & 50896 & 6.94 & $\mathrm{WN} 4 \mathrm{~b}$ & SB1? & multi-variable $e^{b, d}$ \\
\hline 21 & 90657 & 9.80 & WN5o+O4-6 & SB2 & \\
\hline 133 & 190918 & 6.70 & WN5o+O9I & SB2 & in cluster core \\
\hline 138 & 193077 & 8.10 & WN5o+B: & SB2 & \\
\hline 139 & 193576 & 8.10 & $\mathrm{WN} 5 \mathrm{o}+\mathrm{O} 6$ & SB2 & \\
\hline 157 & 219460 & 9.91 & WN5o (+B1II) & visual & in cluster core \\
\hline 24 & 93131 & 6.49 & WN6ha & $?$ & var. light ${ }^{b}$ \\
\hline 25 & 93162 & 8.17 & WN6ha & $?$ & x-ray bright ${ }^{c}$ \\
\hline 134 & 191765 & 8.23 & WN6b & SB1? & \\
\hline 136 & 192163 & 7.65 & WN6b(h) & SB1? & \\
\hline 155 & 214419 & 8.75 & WN6o+09II/Ib & SB2 & \\
\hline 22 & 92740 & 6.44 & WN7ha+O6-9 ${ }^{e}$ & SB2 & \\
\hline 78 & 151932 & 6.61 & WN7h & $?$ & var. (light, RV) $)^{b}$ \\
\hline 16 & 86181 & 8.43 & WN8h & $?$ & var. (light, RV) \\
\hline 40 & 96548 & 7.85 & WN8h & $?$ & var. (light, RV) ${ }^{g}$ \\
\hline 153 & 211853 & 9.08 & $\mathrm{WN} 6 \mathrm{o} / \mathrm{C}+\mathrm{O}+(\mathrm{O}+\mathrm{O})^{f}$ & SB2+SB2: & multiple \\
\hline 52 & 115473 & 9.98 & WC4 & $?$ & var. light ${ }^{b}$ \\
\hline 111 & 165763 & 8.23 & WC5 & $?$ & \\
\hline 23 & 92809 & 9.71 & WC6 & $?$ & \\
\hline 48 & 113904 & 5.69 & WC6 (+O9I) & $\mathrm{SB} 1+$ visual & multiple \\
\hline 14 & 76536 & 9.42 & WC7 & $?$ & \\
\hline 42 & 97152 & 8.25 & $\mathrm{WC} 7+\mathrm{O} 7 \mathrm{~V}$ & SB2 & \\
\hline 79 & 152270 & 6.95 & $\mathrm{WC} 7+\mathrm{O} 5$ & SB2 & in cluster core \\
\hline 86 & 156327 & 9.73 & WC7 (+B0III-I) & visual & \\
\hline 90 & 156385 & 7.45 & WC7 & $?$ & \\
\hline 137 & 192641 & 8.15 & $\mathrm{WC} 7+\mathrm{OB}$ & SB2 & \\
\hline 140 & 193793 & 7.07 & $\mathrm{WC} 7+\mathrm{O} 4-5$ & SB2 & \\
\hline 11 & $\gamma^{2} \mathrm{Vel}$ & 1.74 & WC8+O8.5III & SB2+visual & multiple \\
\hline 113 & 168206 & 9.43 & $\mathrm{WC} 8+08-9$ & SB2 & \\
\hline 135 & 192103 & 8.36 & WC8 & $?$ & \\
\hline 69 & 136488 & 9.43 & WC9 & $?$ & var. light $^{b}$ \\
\hline 103 & 164270 & 9.01 & WC9 & SB1? & var. light ${ }^{b}$ \\
\hline
\end{tabular}

Notes: ${ }^{a}$ van der Hucht (1999), except as noted; ${ }^{b}$ Balona et al. (1989); ${ }^{c}$ Corcoran (1999); ${ }^{d}$ Robert et al. (1992); ${ }^{e}$ Rauw et al. (1996); ${ }^{f}$ Massey (1981); ${ }^{g}$ Antokhin et al. (1995); ${ }^{h}$ Smith \& Maeder (1989); ${ }^{i}$ van der Hucht et al. (1988).

lines of the WR and OB components can be produced. An example of this kind of information for several binaries was presented at IAU Symposium No 163 ( $c f$. Niemela 1995); and

(d) Periodic infrared variability (dust bursts) are observed in some long period binaries with WC type components. 
With simple statistics, a binary frequency of about $20 \%$ comes out from the above considerations for WR stars in our Galaxy, which is of course meaningless, since we lack information on the binary status of most WR stars.

Another possible exercise in order to estimate the binary frequency of WR stars, is to see how these numbers change if we include information for only those stars for which we may expect to have more complete data about their binary nature. We have compiled in Table 1 information for all WR stars with apparent magnitude $v \lesssim 10$ mag. There are 32 stars included in this table; from these, 14 are spectroscopic binaries with known orbits, 4 are possibly single-lined binaries, 2 are visual binaries. Of these binaries, 3 belong to multiple systems, and 2 are located inside cores of clusters. This makes a binary frequency of over $60 \%(50 \%$ excluding "SB1?"), which compares well with a recent study of binary frequency of O-type stars by Mason et al. (1998), who made an astrometric/spectroscopic survey of the brighter O-type stars.

Of course, 32 stars is still small number statistics, and may be biased for binary stars, which appear brighter. However, Table 1 is probably telling us that the binary channel may be more important than others in the formation of stars with WR spectra.

\section{About binary stars with Wolf-Rayet spectra}

What kind of information can we get from the WR stars in binary systems? The mere fact that a star belongs to a binary system is most important when considering the evolutionary scenario. We also may expect to get some estimates of stellar masses from studies of double-lined binaries. However, this is not straightforward for stars which have spectra with broad emission lines, often showing variable shapes according to the orbital phase, or otherwise. The radial velocity orbits of different emission lines also differ in amplitude and phase, and the resulting stellar mass estimates depend on the particular assumption on which emission line is most representative of the true orbital motion of the star with WR-type spectrum.

In any case most of the spectroscopic binaries with WR components are paired with O-type stars, which is an important piece of evidence. The masses of the O-type stars, however, are another unsolved problem.

We will refer here to some particular binary systems, which we consider to be important in exemplifying the sort of individual objects found among the stars with WR spectra.

\subsection{HD $92740=$ WR 22, an old friend}

20 years ago, at IAU Symposium No. 83, Niemela (1979) presented the first indications from radial velocity variations of spectral lines, that WR 22 probably was a double lined binary, and that the WN component of the system was the more massive (see also Conti, Niemela \& Walborn 1979). In their study of WR 22 Moffat \& Seggewiss (1978) had not detected double lines, but only a diminished amplitude of the radial velocity variations of the upper Balmer hydrogen absorption lines, which they attributed to 'the influence of a companion star', presumed to have higher mass than the WN component.

Recently Rauw et al. (1996) have published a new orbit for the eclipsing system WR 22 from spectra with higher $\mathrm{S} / \mathrm{N}$ and resolution, confirming our pre- 
vious findings. It is important to note that the high mass of the WN component (72 $\mathrm{M}_{\odot}$ according to Rauw et al. ), implies that it must be a H-burning star, probably an overmassive (i.e., near the limit of stability) star from its birth. WR 22 actually appears as the most massive star known in a spectroscopic binary system.

In their three dimensional classification for WN Stars, Smith, Shara \& Moffat (1996, SSM96), introduced the classification of the Carina WN stars, WR 22 among them, as typical of WNha stars, a designation which is applied to WN type spectra with $\mathrm{H}$ emission and intrinsic absorption lines, usually the higher Balmer lines. We note that with $\mathrm{M}_{v} \simeq-7$, these are the brightest WN-type stars, which would dominate in integrated spectra of HII regions, as observed in e.g., NGC 3603 in our Galaxy (Drissen et al. 1995) and in R 136, the central cluster of 30 Dor in the Large Magellanic Cloud (Massey \& Hunter 1998). Few such stars are known in our Galaxy, and here we will show spectral observations which add two new members to this class.

\subsection{WR 29, a new WN7ha+OB binary}

The above mentioned paper (SSM96) also includes an interesting table (their Table 8), with estimates of relative brightness of companions to the WN stars based on observed and expected EW of He II $\lambda 5411 \AA$ emission in the spectra. However, not all stars in this table are known binaries. In particular WR 29 is classified as WN7h+abs, which means that absorption lines of unknown origin are present in the spectrum.

We have obtained moderate resolution CCD spectra of WR 29 in 1997-8 with the Cassegrain spectrograph attached to the $2.1 \mathrm{~m}$ telescope at CASLEO, San Juan, Argentina. Figure 1 shows a spectrum of WR 29, together with that of WR 22 for comparison.

The first observations obtained during successive nights, already revealed considerable radial velocity variations of the emission lines, sign of a short period binary system. A period-search routine applied to the emission line radial

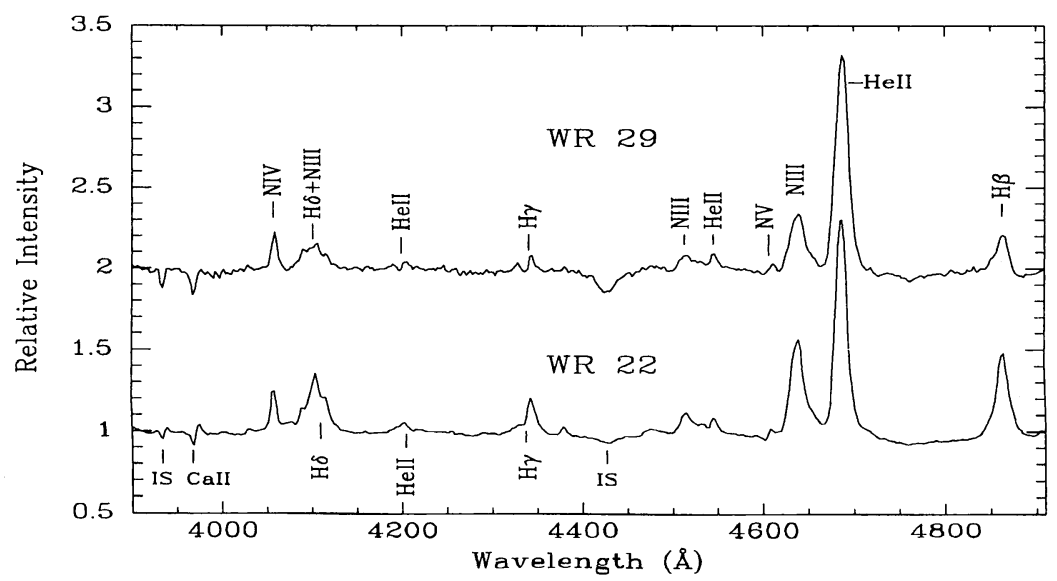

Figure 1. Blue spectra of WR 29 and WR 22 (CASLEO March 1997). 

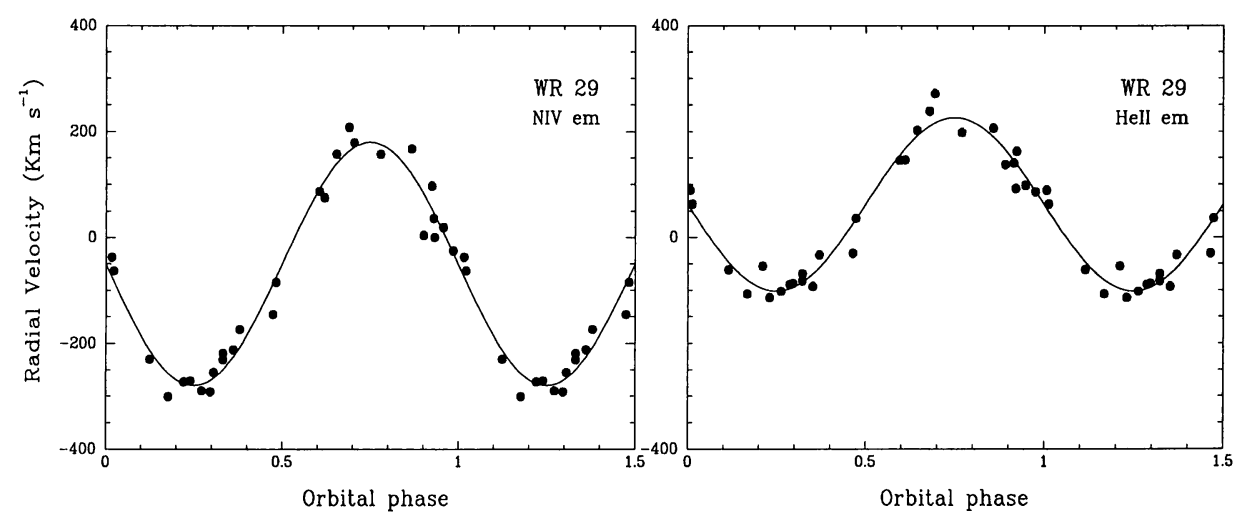

Figure 2. Radial velocity variations of the N IV and He II emission lines in the spectrum of WR 29 , folded with $P=3.146 \mathrm{~d}$.

velocities, produces as a best period 3.164 days. However, the radial velocities of the absorption lines are difficult to measure in our spectra. It is obvious that the WN component has intrinsic blueshifted absorption components of the emission lines, which blend with the absorptions of the OB companion, particularly when the OB star has its most negative velocity.

Figure 2 shows the radial velocity variations of the $\mathrm{N}$ IV $\lambda 4058 \AA$ and He II $\lambda 4686 \AA$ emissions in the spectrum of WR 29 phased with the period of 3.164 days. Preliminary orbital elements are published by Niemela \& Gamen (1999). Higher spectral resolution is necessary to determine the radial velocity orbit of the OB companion.

\section{3. $\quad$ HD $65865=$ WR 10 , the only galactic WN5ha(+A-type visual companion)}

WR 10 has been cited throughout most of the literature as $\mathrm{WN}+(\mathrm{OB}$ visual companion). It is in fact a close visual double, the WR star being the brighter northern component.

Figure 3 shows a CCD spectrum of the fainter companion of WR 10, obtained at CASLEO. There are no He lines in this spectrum, only broad $\mathrm{H}$ Balmer absoprtions and some metallic lines. Therefore it is not of type OB, but of spectral type $\sim$ A2V. Probably it is not physically bound to WR 10 .

A previous version of this result, based on photographic spectra, was presented many years ago (Niemela 1981) in a Latin American Astronomy Meeting, as noticed by van der Hucht et al. (1988).

On the other hand, the spectrum of WR 10, classified by SSM96 as WN5h, is illustrated in Figure 4, where absorption lines of the same type as in the spectra of the Carina WN stars are seen.

About 20 spectra of WR 10 obtained at CTIO in 1980, and at CASLEO in 1997-98, do not show radial velocity variations beyond the errors of measurement. The higher Balmer absorption lines appear only slightly, if at all, blueshifted from their normal position. WR 10 seems to be a genuine WN5ha type star, thus far the only one known in our Galaxy. 


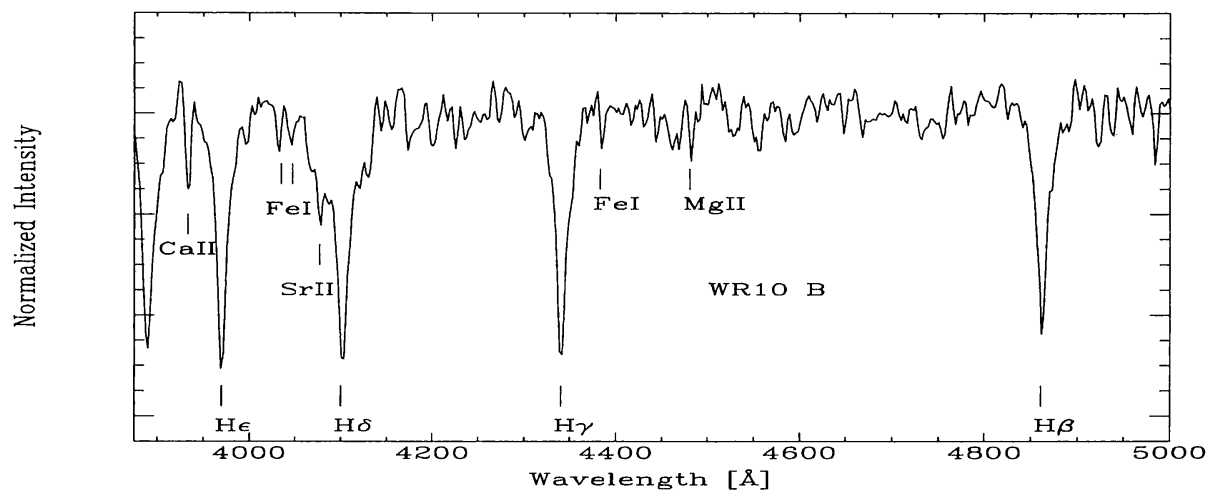

Figure 3. A blue spectrum of the fainter SW component of WR 10 (CASLEO, March 1997).

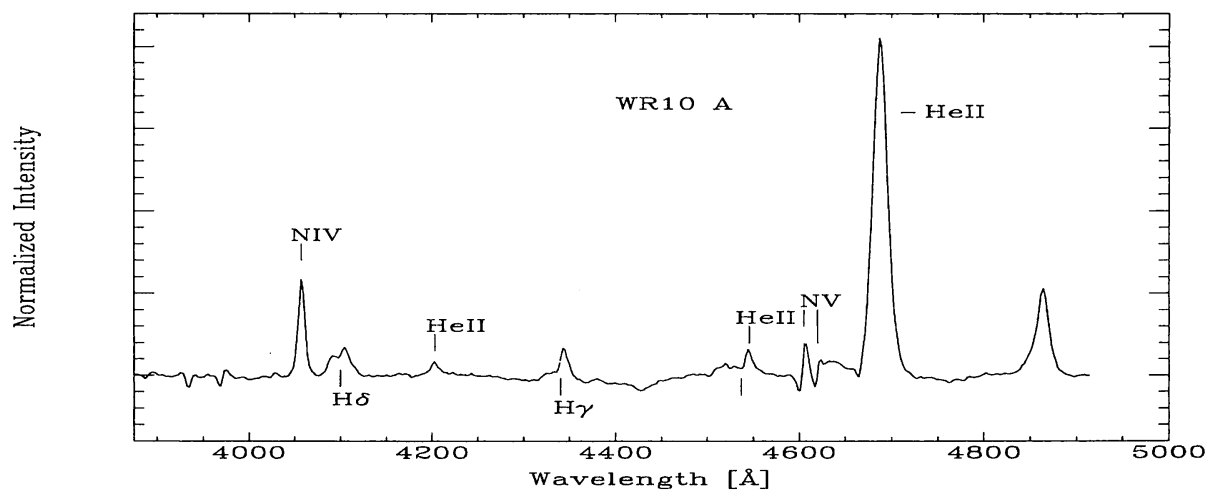

Figure 4. Blue optical spectrum of WR 10 obtained at CASLEO

\section{Wolf-Rayet binaries in the Small Magellanic Cloud}

WR stars in the Small Magellanic Cloud are few in number: only nine are known to date (Azzopardi \& Breysacher 1979; Morgan et al. 1991). Spectroscopic orbits are known for three of them and radial velocity variations are known for another two.

\subsection{HD 5980, with friends like this ...}

The most spectacular recent episode in WR binaries has been the eruption of the luminous WR binary within the brightest $\mathrm{H}$ II region of SMC, namely the eclipsing binary HD 5980. There was a special session on this binary during the Kona Workshop on Luminous Blue Variables (Nota \& Lamers 1996), and yesterday again a special session on HD 5980 was held.

We note that HD 5980 may consist of two massive WNha type stars, because the absorption lines present in the spectrum before the eruption were blueshifted with respect to the nebular emission, and only showed scatter (Niemela et al. 


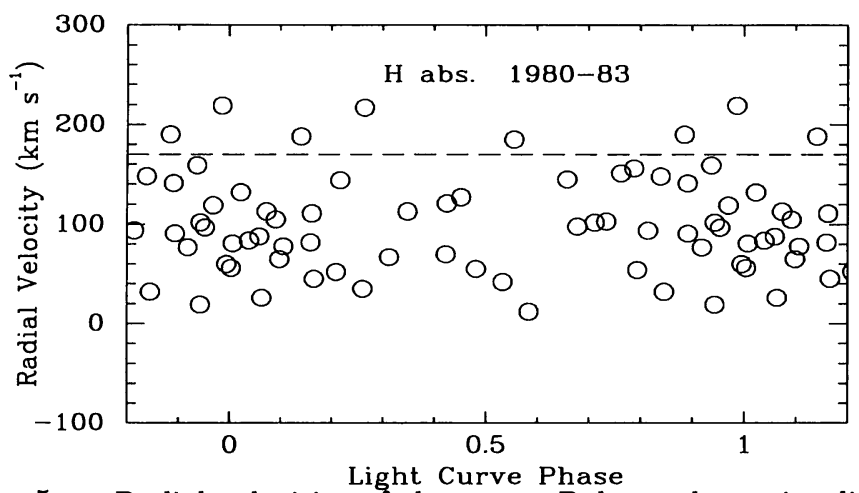

Figure 5. Radial velocities of the upper Balmer absorption lines in the spectrum of HD 5980 before the eruption. Nebular velocity is indicated by a dashed line.

1997), or dubious variations (Moffat et al. 1998), when measured for determination of radial velocities. This is what would be observed if both binary components had blue-shifted absorption lines not separated due to poor spectral resolution. This is illustrated in Figure 5, where we show the radial velocities of the upper Balmer absorption lines observed in the spectrum of HD 5980 before the eruption, folded in the binary period (a similar figure was shown by Niemela et al. (1997)).

\subsection{SMC/AB 7, another massive WR binary}

AB 7 (Azzopardi \& Breysacher 1979), located inside the second brightest H II region in the SMC, has been shown to have variable radial velocity by Moffat (1988). The blue optical spectrum of AB 7, illustrated in Figure 6 is dominated by the strong emission line of He II $\lambda 4686 \AA$. Absorption lines from a companion star are moving with opposite orbital motion, as shown previously by Niemela (1994).

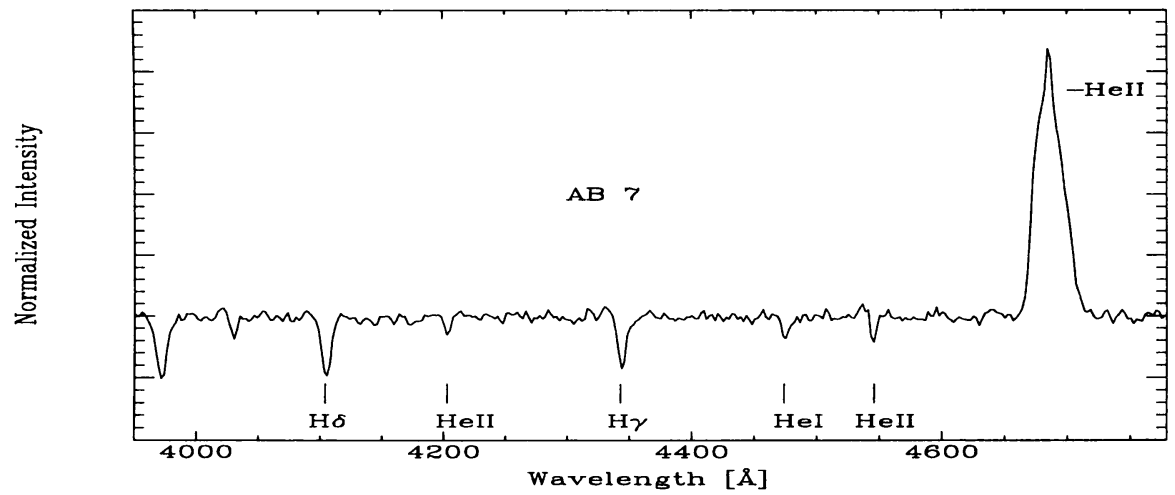

Figure 6. Blue optical spectrum of $\mathrm{AB} 7$ obtained at CASLEO 


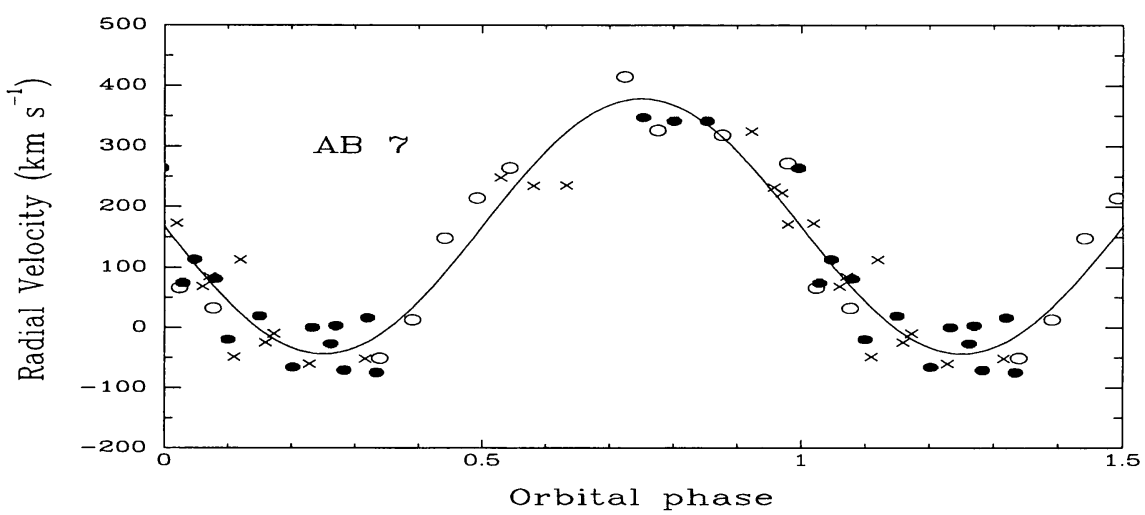

Figure 7. Radial velocities of the He II $\lambda 4686 \AA$ emission in the spectrum of $\mathrm{AB} 7$ folded in the period of 19.64 days. Crosses represent photographic data, open circles, 2Df-CTIO data and filled circles, CASLEO-CCD spectra.

We have obtained optical spectra of AB 7 at CTIO between 1985 and 1990, and at CASLEO after 1992. Our CCD spectra obtained at CASLEO indicate a spectral classification of $\mathrm{O} 6 \mathrm{~V}$ : for the $\mathrm{OB}$ component. The WN component is difficult to classify, since only He II emission lines are observed. According the criteria for galactic and LMC WN stars (SSM96), the spectral type could be WN2.

Radial velocities of the strong He II $\lambda 4686 \AA$ emission line from our spectra combined with published data (Moffat 1988), allowed us to determine a binary period of 19.56 days for AB 7 (almost equal to that of HD 5980!). In Figure 7 we show the radial velocity variations of He II emission for different data sets folded in the period of 19.56 days.

The radial velocity variations of the He II emission and the absorption lines folded in this period indicate a circular orbit, within the observational errors. These radial velocity curves imply that $\mathrm{AB} 7$ is a massive binary system, with

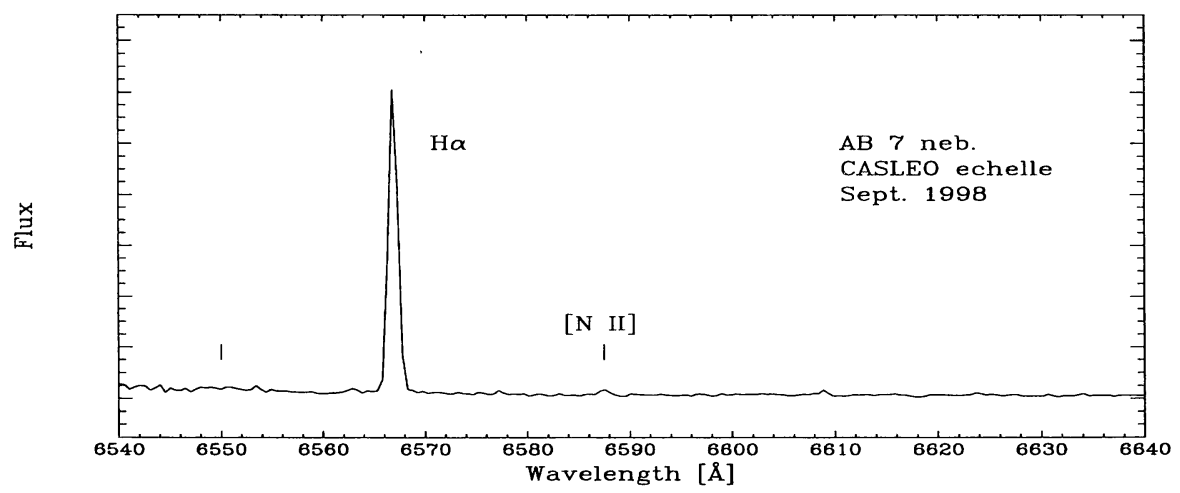

Figure 8. Spectrum of the $\mathrm{H} \alpha$ region of the nebula close to $\mathrm{AB} 7$. 
minimum masses of 40 and $20 \mathrm{M}_{\odot}$ for the $\mathrm{O} 6$ and the WN components, respectively (Niemela \& Morrell 1999).

AB 7 is also the exciting star of a very high ionization gas bubble showing nebular He II $\lambda 4686 \AA$ emission. High resolution spectra, obtained at CASLEO in 1998, September, of the $\mathrm{H} \alpha$ region of the nebula close to the star, show only traces of nebular [NII], as seen in Figure 8, indicating that there is no detectable $\mathrm{N}$ enhancement.

The nebular environment of AB 7 may also be considered as indicative of young age, since the stellar winds have not yet had time to blow the gas away.

\section{Other old friends}

After the surprising eruption of HD 5980, an obvious question arises: how many of the other binary systems with WR-type components may show a similar behaviour?

With this question in mind, we have started at CASLEO an observing program of WR binaries, reobserving known binary systems. As preliminary results, new orbits for WR 21 and CV Ser are shown.

\subsection{HD $90657=$ WR 21, new data of an old friend}

This WN5o+O4-6 binary system was observed by Niemela \& Moffat in 1982 . We have obtained CCD spectra of WR 21 at CASLEO in 1997-98. With the new radial velocities of the emission lines from these spectra, we have improved the binary period to $P=8.2545$ days. The new He II $\lambda 4686 \AA$ and $N$ V $\lambda 4603 \AA$ emission line orbits agree with the previous observations. However, the new orbit for N IV $\lambda 4057 \AA$ emission shows a systemic velocity which is considerably more negative than the one derived from previous observations. This is illustrated in Figure 9.

Keeping in mind the strange behaviour of the N IV emission line in the spectrum of HD 5980, WR 21 is worthy of close follow-up observations.
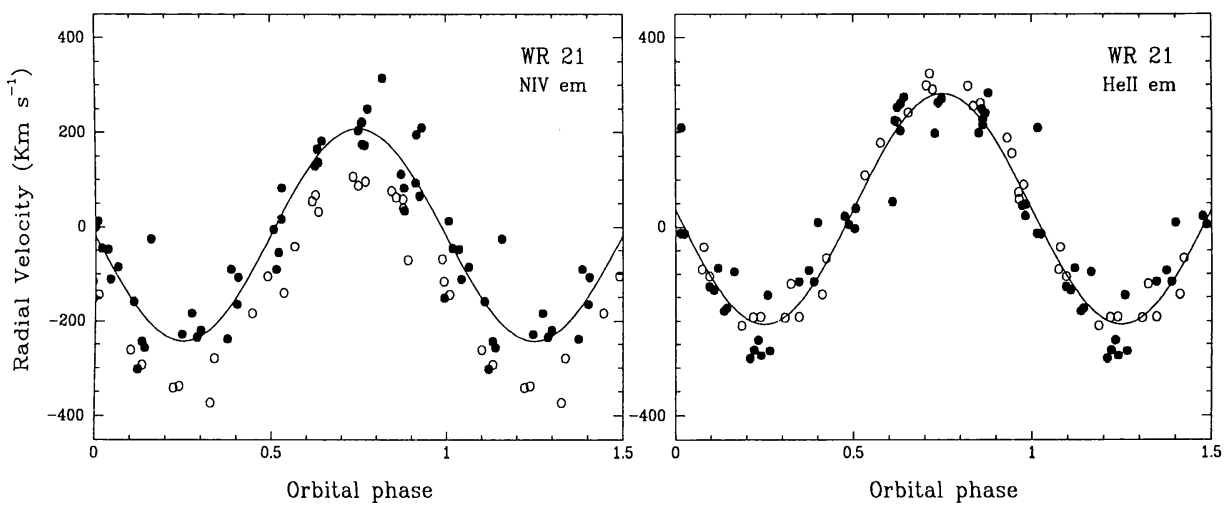

Figure 9. N IV and He II emission line orbits for WR 21 folded in the new period of 8.2545 days. The new 1997-8 observations are indicated as open symbols, and the previous data as filled symbols. 


\section{2. $\quad$ CV Ser $=$ WR 113, a new orbit of another old friend}

CV Ser, classified as WC8+O8-9, is famous for the episode of eclipses which have disappeared. We have obtained new optical spectra of this star, and considering that the absorption lines of the O-type component should allow us to obtain a more stable orbital solution than the emissions, have based our orbital determination on the mean velocities of the absorption lines. We find that the orbit is elliptical, instead of the previously assumed circular shape. The new orbital fit for the absorption lines is illustrated in Figure 10 along with the radial velocities of He II $\lambda 4686 \AA$ emission. Orbital elements corresponding to these observations have been published by Niemela et al. (1996).

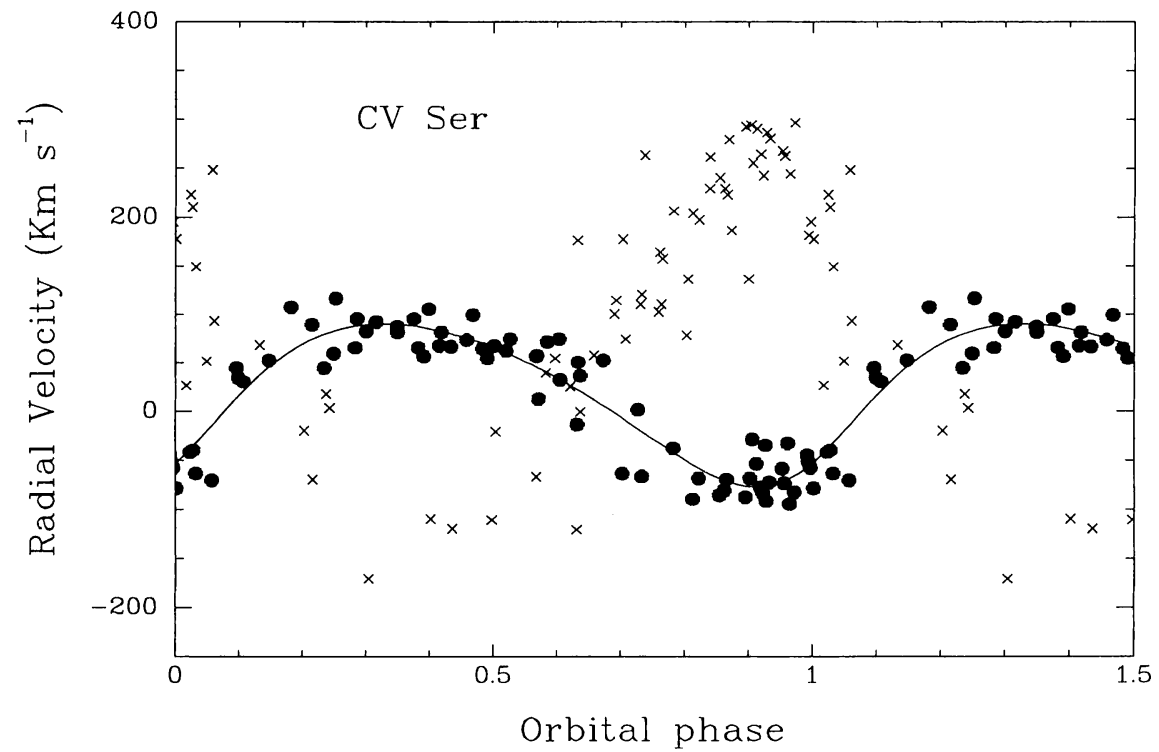

Figure 10. Radial velocity orbits for the binary components of CV Ser. Filled circles correspond to mean velocities of the absoption lines of the $\mathrm{O}$ type star, and the crosses to the He II emission of the WC component.

\section{Conclusions}

The masses of stars with WR spectra span a wide range. In particular, the binary systems with WR components of high luminosity have also high masses; therefore it seems that both $\mathrm{H}$ burning and He burning stars can appear with WR-type spectra. This in turn means that it would probably be more appropriate to talk about WR phenomena in different stars. However, more observations are needed!

Acknowledgments. We are grateful to the directors and staff of CTIO and $C A S L E O$ for the use of their facilities. We acknowledge the use, at CASLEO, of the CCD and data acquisition system supported through US NSF grant AST90-15827 to R.M. Rich. We are indebted to R. Barbá for help with figures. We 
wish to thank the referee, Dr. Tony Moffat, for his interesting comments and help in improving the English of this paper.

\section{References}

Antokhin, I., Bertrand, J.-F., Lamontagne, R., Moffat, A.F.J., Matthews, J. 1995, AJ 109,817

Azzopardi, M., Breysacher, J. 1979, A\&A 75, 120

Balona, L.A., Egan, J., Marang, F. 1989, MNRAS 240, 103

Conti, P.S., Niemela, V.S., Walborn, N.R. 1979, ApJ 228, 206

Corcoran, M.F. 1999, in: N.I. Morrell, V.S. Niemela \& R. Barbá (eds.), Hot Stars in Open Clusters of the Galaxy and the Magellanic Clouds, RevMexAA-SC in press

Drissen, L., Moffat, A.F.J., Walborn, N.R., Shara, M.M. 1995, AJ 110, 2235

Dougherty, S.M., Williams, P.M., van der Hucht, K.A., Bode, M.F., Davis, R.J. 1996, MNRAS 280, 963

van der Hucht, K.A. 1999, in preparation

van der Hucht, K.A., Conti, P.S., Lundström, I., Stenholm, B. 1981, SSR 28, 227

van der Hucht, K.A., Hidayat, B., Admiranto, A.G., Supelli, K.R., Doom, C. 1988, A\&A 199, 217

Mason, B.D., Gies, D.R., Hartkopf, W.I., Bagnuolo, W.G., Brummelaar, T.T., McAlister, H.A. 1998, AJ 115, 821

Massey, P. 1981, ApJ 244, 157

Massey, P., Hunter, D.A. 1998, ApJ 493, 180

Moffat, A.F.J. 1988, ApJ 330, 766

Moffat, A.F.J., Marchenko, S.V., Bartzakos, P., Niemela, V.S., Cerruti, M.A., Magalhães, A.M., Balona, L., St-Louis, N., Seggewiss, W., Lamontagne, R. 1998, ApJ 497, 896

Morgan, D.H., Vassiliadis, E., Dopita, M.A. 1991, MNRAS 251, 51P

Niemela, V.S. 1979, in: P.S. Conti \& C. de Loore (eds.), Mass Loss and Evolution of O-type Stars, Proc. IAU Symp. No. 83 (Dordrecht: Reidel), p. 291

Niemela, V.S. 1981, RevMexAA 6, 244

Niemela, V.S. 1995, in: K.A. van der Hucht \& P.M. Williams (eds.), Wolf-Rayet Stars: Binaries, Colliding Winds, Evolution, Proc. IAU Symp. No. 163 (Dordrecht: Kluwer), p. 223

Niemela, V.S., Barbá, R.H., Morrell, N.I., Corti, M. 1997, in: A. Nota \& H. Lamers (eds.), Luminous Blue Variables: Massive Stars in Transition, ASP-CS 120, 222

Niemela, V.S., Gamen, R. 1999, in: N.I. Morrell, V.S. Niemela \& R. Barbá (eds.), Hot Stars in Open Clusters of the Galaxy and the Magellanic Clouds, RevMexAA-SC in press

Niemela, V.S., Moffat, A. F. J. 1982, ApJ 259, 213

Niemela, V.S., Morrell, N.I. 1999, in: Y.-H. Chu, N.B. Suntzeff, J.E. Hesser, \& D. Bohlender (eds.), New Views of the Magellanic Clouds, Proc. IAU Symp. No 190 (San Fransisco: ASP), in press

Niemela, V.S., Morrell, N.I., Barbá, R.H., Bosch, G.L. 1996, in: V.S. Niemela \& N.I. Morrell (eds.), Colliding Winds in Binary Stars, RevMexAA-SC 5, 100

Niemela, V.S., Shara, M.M., Wallace, D.J., Zurek, D.R., Moffat, A.F.J. 1998, AJ 115, 2047

Nota, A., Lamers, H. (eds.), Luminous Blue Variables: Massive Stars in Transition, ASP-CS 120 
Rauw, G., Vreux, J.-M., Gosset, E., Hutsemekers, D., Magain, P. Rochowicz, K. 1996, A\&A 306, 771

Robert, C., Moffat, A.F.J., Drissen, L., Lamontagne, R., Seggewiss, W., Niemela, V.S., Cerruti, M.A., Barrett, P., Bailey, J., Garcia, J., Tapia, S. 1992, ApJ 397, 277

Smith, L.F., Maeder, A. 1989, A\&A 211, 71

Smith, L.F., Shara, M.M., Moffat, A.F.J. 1996, MNRAS 281, 163

Williams, P.M., Dougherty, S.M., Davis, R.J., van der Hucht, K.A., Bode, M.F., Setia Gunawan, D.Y.A. 1997, MNRAS 289, 10

\section{Discussion}

Crowther: WR 10 (HD 65865) represents a remarkable galactic object since it is spectroscopically identical to the H-rich luminous WN stars in R 136a. The non-binary nature for WR 10 is therefore a very important result.

Langer: From binary models, I would expect some systematical differences between the properties of binaries with WN stars compared to those with WC stars. (e.g., concerning the periods). Have you searched for such effects in your data?

Niemela: The number of known binaries is still small, and the shorter periods are much easier to find. There are short and long period binaries in both WR sequences.

Vanbeveren: As far as WR single stars are concerned, one has to wonder whether a WR star is a real single star or a WR star with a binary history (a descendant of a mass gainer in a close binary where the supernova explosion of the primary disrupted the system). The evolution of the latter may be completely different from the evolution of a normal single star.

Leitherer: My question comes in three parts: (1) What are the prospects for detecting late-type companions around WR stars? (2) What is the percentage of known WR systems with a RSG component? (3) Can this percentage be used to place constraints on models to produce WR stars from RSG's?

Niemela: As far as I know, there are no RSG companions among the WR binaries in our Galaxy (However, see the comment of Walborn, below).

Walborn: HDE 269229, a WN9 object in the LMC, is a spectrum composite with a red supergiant, although no radial-velocity variations have been observed to my knowledge.

Moffat: With only nine WR stars, the SMC is statistically not a good place to talk about the binary frequency. However, given that stellar models predict that $\sim 100 \%$ of the SMC WR stars must be binary since the $0.1 \mathrm{Z}_{\odot}$ cannot lead to single-star WR formation via stellar wind, it might be more pertinent to look for non-binaries!

Niemela: Yes, but it is difficult if not impossible to show that a star is not a binary, since you may always have a too long period, too low orbital inclination, etc. 